\title{
Acute monoarticular arthritis caused by Maltese cross-like crystals
}

A 44-year-old woman who was previously healthy was seen in the emergency department with a 1-day history of increasing pain in her left knee. She denied trauma and reported no family history of arthritis. Her temperature was $38.5^{\circ} \mathrm{C}$, and her left knee was swollen, warm and tender, but no erythema was noted. Aspiration was performed, and $55 \mathrm{~mL}$ of thin, cloudy fluid was obtained. The fluid showed a neutrophil count of $49.5 \times 10^{9} / \mathrm{L}$ and an erythrocyte count of $6.5 \times$ $10 \% /$ L. Blood and synovial fluid was obtained for culture, and intravenous therapy with cefazolin (1.5 $\mathrm{g}$ every 8 hours) was started. The Gram stain of the fluid showed more than 100 neutrophils per low-power field, but no organisms were seen, and the cultures of blood and synovial fluid were sterile. Use of a polarizing microscope failed to reveal urate or calcium pyrophosphate crystals. However, many birefringent Maltese cross-like spherules were seen in the synovial fluid (Fig. 1). The spherules varied in size from under $1 \mu$ up to $10 \mu$. They were present both as free spherules and within the cytoplasm of neutrophils. Some neutrophils had several intracytoplasmic spherules, which suggested that they had been phagocytosed (Fig. 2, arrows). Because these spherules are thought to be composed of lipids, both serum and synovial fluid were analyzed for cholesterol and triglycerides. The cholesterol concentratrion was $4.3 \mathrm{mmol} / \mathrm{L}$ in the serum sample and $1.8 \mathrm{mmol} / \mathrm{L}$ in the syn$\infty$ ovial fluid; the triglyceride values were 1.18 and $0.29 \mathrm{mmol} / \mathrm{L}$ respectively.

The antibiotic therapy was stopped after the second dose, and the patient was discharged with a prescription for oral indomethacin therapy $(25 \mathrm{mg} 3$ times daily). However, she returned to the outpatient clinic 5 days later, at which time $90 \mathrm{~mL}$ of fluid was removed from her knee, but no samples were sent for further analysis. The knee was injected with $40 \mathrm{mg}$ of methylprednisolone, and the indomethacin dose was increased. The patient's knee was significantly improved at follow-up 8 days later.

The most common causes for acute monoarticular arthritis are infection and crystals. Of the infectious agents, Staphylococcus aureus and Neisseria gonorrboeae are the most frequently isolated. ${ }^{1}$ Both gout and pseudogout are also capable of producing acute arthritis. It is difficult to differentiate the causes of acute monoarticular arthritis by clinical findings alone. Gram staining and culture as well as a search for crystals with a polarizing microscope should be requested on synovial fluid. In the case we have described, we were unable to find an infectious agent or any evidence of the usual crystals seen in gout or pseudogout. However, we did find many crystals in the synovial fluid that exhibited a Maltese crosslike birefringence. A PubMed search using the terms "Maltese cross arthritis," "acute arthritis associated with birefringent crystals" and "lipid microspherule arthritis" as well as a search of references from retrieved articles for additional cases resulted in 13 other cases being found, with the first reported in 1980. Ten of these cases also had monoarticular involvement, and slightly more women than men were affected (8 v. 5). The patients' ages ranged from 14 to 63 years. The leukocyte count in the synovial fluid varied from 6.0 to $120.0 \times 10^{9} / \mathrm{L}$.

Although these crystals are composed of lipids, patients have seldom had abnormal lipid concentrations in serum or synovial fluid samples. ${ }^{2}$ Lipid spherules can be formed from fat either released into synovial fluid or as a result of supersaturation. Trauma causing hemarthrosis has been associated with lipid droplet formation. ${ }^{3}$ The

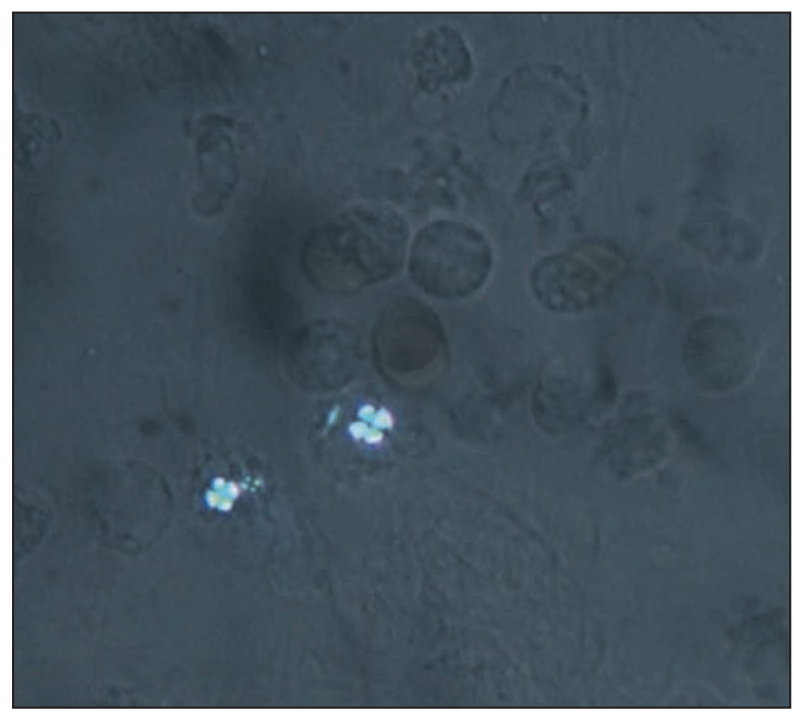

Fig. 1: Polarization microscopy of synovial fluid in woman with acute monoarticular arthritis. Three intracytoplasmic spherules are seen, each with a birefringent Maltese cross-like appearance. Magnification $\times 400$.

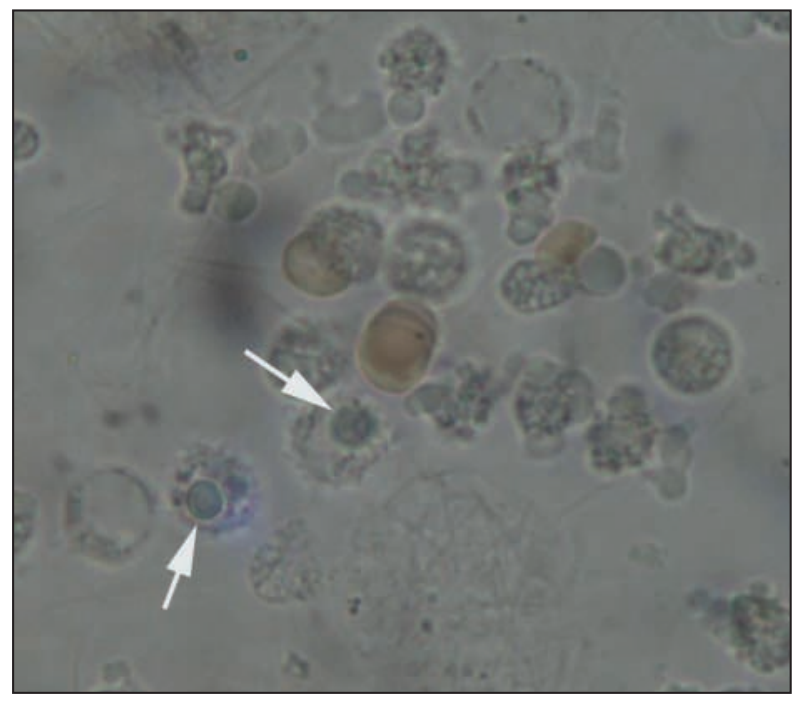

Fig. 2: Unstained smear of synovial fluid (same field and magnification as in Fig. 1). Two polymorphonuclear cells are seen, each with a large intracytoplasmic inclusion (arrows). A few erythrocytes are also present. 
Practice lipid spherules have also been seen in experiments in which autologous erythrocytes injected into rabbit knee joints induced an acute inflammatory arthritis. Another theory suggests the breakdown of cell membrane phospholipids as the source of the crystals.

Regardless of the pathogenesis of the crystals, their presence in synovial fluid is strongly associated with clinical symptoms such as pain and fever. As well, many of the crystals can be seen intracellularly. Treatment is usually suc- cessful with either colchicine or an NSAID. To our knowledge, our case was the first in which intra-articular steroid injection was used.

Joe Dylewski

Director of Laboratories

St. Mary's Hospital

Montréal, Que.

Zuhier Awan

Resident in Laboratory Medicine

McGill University

Montréal, Que.

Indrojit Roy

Division of Pathology

St. Mary's Hospital

Montréal, Que.

\section{References}

1. Siva C, Velazquez C, Mody A, Brasington R. Diagnosing acute monoarticular arthritis in adults: a practical approach for the family physician. Am Fam Physician 2003;68:83-90.

2. Gardner GC, Terkeltaub RA. Acute monoarthritis associated with intracellular positively birefringent Maltese cross appearing spherules. $f$ Rheumatol 1989;16:394-6.

3. Gregg J, Nixon JE, Di Stefano V. Neutral fat globules in traumatized knees. Clin Ortho 1978;132:219-24.

4. Choi SJ, Schumacher HR, Clayburne G. Experimental haemarthrosis produces mild inflammation associated with intracellular Maltese crosses. Ann Rheum Dis 1986;45:1025-8.

5. Choi SJ, Schumacher HR, Clayburne G, Rothfuss SM, Sieck M. Liposomeinduced synovitis in rabbits. Arthritis Rheum 1986;29:889-96.

\section{P HYSICIAN \\ MA N A G E R I NSTITUTE}

$20055 \quad P$ I S C H E D U L E

A five level credit program exclusively for physicians designed to develop superior leadership and management skills

Approved for RCPSC, CFPC, CCHSE credits

\section{In-house PMI}

A practical, cost effective and focused training opportunity held on-site for medical leaders and managers

For information:

tel $800663-7336$ or $613731-8610$

X2319 (PMI) or X2261 (In-house PMI)

professional_development@cma.ca

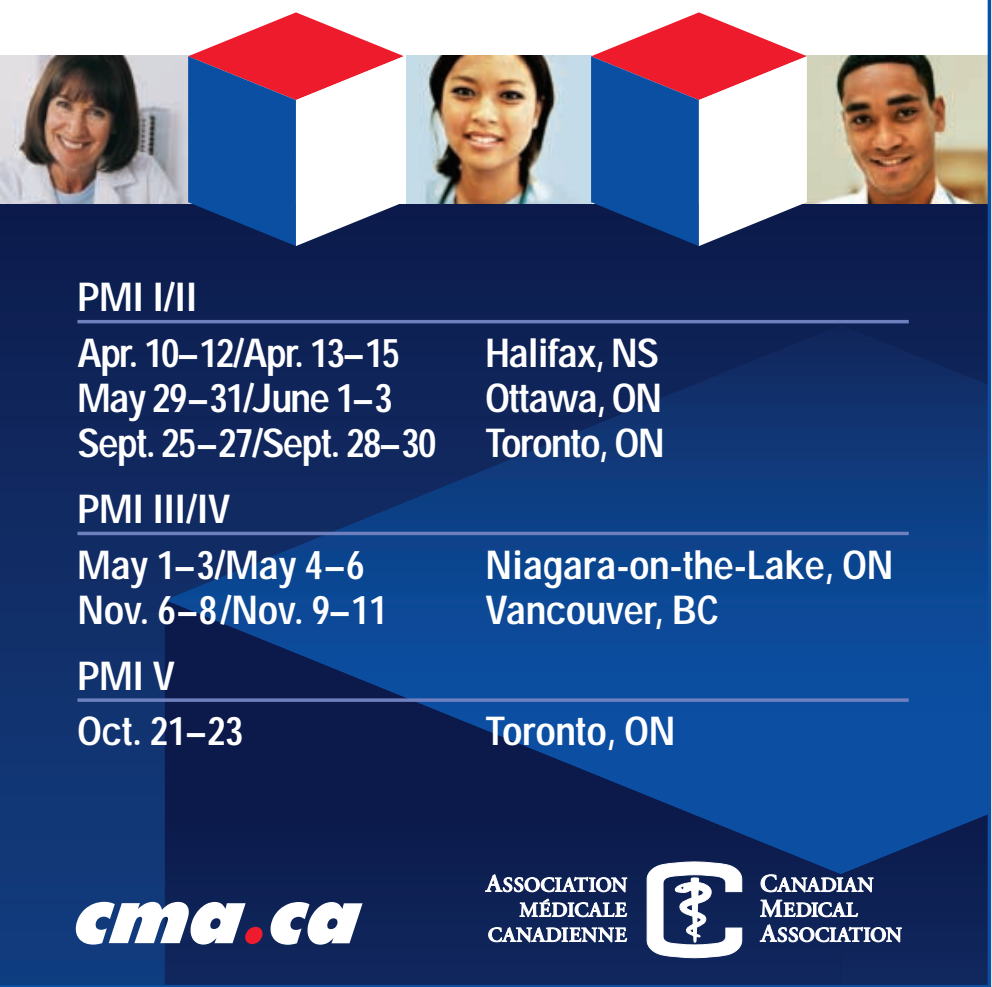

